\title{
The knowledge of Polish primary care physicians about bariatric surgery
}

\author{
Piotr Major ${ }^{1}$, Tomasz Stefura², Monika Jezierska-Kazberuk ${ }^{3}$, Michat Wysocki ${ }^{2}$, Michał Pędziwiatr ${ }^{1}$, \\ Magdalena Pisarska1, Piotr Małczak ${ }^{1}$, Artur Kacprzyk², Andrzej Budzyński² \\ ${ }^{1} 2^{\text {nd }}$ Department of General Surgery, Jagiellonian University Medical College, Krakow, Poland \\ ${ }^{2}$ Students' Scientific Group at $2^{\text {nd }}$ Department of General Surgery, Jagiellonian University Medical College, Krakow, Poland \\ ${ }^{3}$ SCDZ Medicina, Krakow, Poland
}

Videosurgery Miniinv 2016; 11 (3): 164-170

DOI: $10.5114 /$ wiitm.2016.62446

\begin{abstract}
Introduction: The general practitioner $(G P)$ can play a key role in this multi-disciplinary team, coordinating care provided by dietitians and surgeons, maximizing the potential benefits of surgery. Therefore, it seems important to verify changes in GPs' knowledge about surgical treatment of obesity.

Aim: To reassess knowledge of obesity surgical treatment among Polish primary care physicians and their willingness to improve it in the future.

Material and methods: To assess the knowledge of Polish primary care physicians about surgical treatment of obesity, a prospective study, which included an anonymous online questionnaire, was conducted in the years 2015-2016. Results: Two hundred and six physicians answered the invitation. One hundred and sixty-six (81.8\%) respondents were familiar with the indications for bariatric operation. The great majority of respondents, 198 (96.6\%), were aware that bariatric surgery is efficient in the treatment of the metabolic syndrome. The study revealed a disproportion between the number of patients who would be potential candidates for bariatric treatment, who are currently under care of participating physicians, and the number of patients who are referred to a bariatric surgeon.

Conclusions: Our study demonstrates that nowadays bariatric surgery is a recognized method of treatment, but physicians remain reluctant to refer their patients for surgical treatment of obesity. It was found that there is a large disproportion between the number of patients who are referred to a bariatric surgeon and the number of patients who require this treatment. It may be a result of lack of knowledge in the field of bariatric surgery.
\end{abstract}

Key words: bariatric surgery, knowledge, general physicians.

\section{Introduction}

According to the World Health Organization, overweight and obesity are becoming a growing health threat, replacing more traditional concerns, which include malnutrition and infectious diseases, as one of the most significant health issues [1]. Obesity is referred to as an epidemic disease [2]. In addition, obesity prevalence in children has been growing rapidly year by year [3]. The wide range of obesity-associated comorbidities (such as systemic arterial hypertension, type II diabetes mellitus, hypertriglyceridemia, sleep apnea, coronary disease and stroke) makes it a significant issue in modern medicine [4-6].

Obesity treatment involves dietary approaches, lifestyle intervention, pharmacotherapy, endoscopic intragastric balloon and surgery $[7,8]$. However,

\section{Address for correspondence}

Piotr Major MD, PhD, 2 $2^{\text {nd }}$ Department of General Surgery, Jagiellonian University Medical College, 21 Kopernika St, 31-501 Krakow, Poland, phone: +48 693313 948, e-mail: majorpiotr@gmail.com 
bariatric surgery, in comparison with non-surgical treatment, has been proven to have better outcomes in terms of body mass index (BMI) reduction and remission of several comorbidities associated with obesity [9-12]. It is important to emphasize that surgical treatment of obesity can spectacularly improve quality of life, especially in morbidly obese patients [13]. Therefore, bariatric operations are performed more often [14]. The most commonly performed bariatric procedures are laparoscopic Roux-en-Y gastric bypass (LRYGB) and laparoscopic sleeve gastrectomy (LSG) $[15,16]$.

Obesity should be approached similarly to a complex chronic disease, which requires multi-disciplinary care and a high degree of patient's participation [17]. It seems that the general practitioner (GP) can play a key role in this multi-disciplinary team, coordinating care provided by dietitians and surgeons, maximizing the potential benefits of surgery [18]. Patients rely on their GPs to verify the information which they found via the internet, and GPS remain the most influential people when it comes to choosing the surgeon or hospital for bariatric surgery [19]. According to a study conducted between 2010 and 2011 in Poland, GPs were not able to identify patients for bariatric procedures, although most of them were familiar with the term 'bariatrics' [20]. It would seem that a lot of physicians were not considering bariatric surgery as a therapeutic option for their patients [21]. Since 2011, an extensive education concerning surgical treatment of obesity has been introduced among students, surgeons and specialists in other medical disciplines. Therefore, it seems important to verify changes in GPs' knowledge about surgical treatment of obesity.

\section{Aim}

The objective of our study was to reassess knowledge of obesity surgical treatment among Polish primary care physicians and their willingness to improve it in the future.

\section{Material and methods}

A prospective study, which included an anonymous online questionnaire, was conducted in the years 2015-2016. The questionnaire included multiple-choice and open questions. It comprised 3 parts. The first part of the survey, consisting of 4 questions, was designed to obtain information about the physician: gender, age, specialization, current area of employment. The second part consisted of 7 questions, which were supposed to assess knowledge about surgical treatment of obesity. Question 5 evaluated awareness of the possibility of obesity surgical treatment, question 6 knowledge about the indications for a bariatric procedure, question 7 and question 8 the information regarding the most common bariatric operations and how they are carried out, question 9 knowledge about metabolic surgery financing in Poland, and question 10 and question 11 about the role of bariatrics in treatment of metabolic syndrome. The third part of the survey included 4 questions designed to evaluate current principles of care for obese patients and willingness to expand GPs' knowledge about bariatric surgery. In question 12 physicians were asked whether they had ever referred their patient to a bariatric surgeon. Question 13 estimated the number of patients who are being referred to a bariatric surgeon by survey respondents. Question 14 focused on the number of GPs' patients who would be candidates for bariatric surgery. Question 15 assessed their willingness to improve their knowledge in the future (Appendix).

The study was performed in accordance with the ethical standards laid down in the 1964 Declaration of Helsinki and its later amendments.

Two hundred and six physicians answered the invitation to take part in the survey. Two hundred answered all questions. One hundred and thirtynine $(67.48 \%)$ respondents were female and 67 (32.52\%) were male. Mean age in the study group was $46.39 \pm 10.87$ years (male: $48.45 \pm 11.74$, female: $45.41 \pm 10.28)$. Most of the physicians were from the Małopolska voivodeship (44; $21.36 \%$ ) (Table I). The most common specialization was general practitioner - 174 (84.9\%) respondents. The second most common was internal medicine - 13 (6.3\%). Nine (4.4\%) respondents were pediatricians and 9 (4.4\%) had other specializations (radiology, ophthalmology, endocrinology, palliative care, occupational medicine, public health, geriatrics) or had no specialization.

\section{Results}

Only 1 (0.5\%) physician was not familiar with the possibility of surgical treatment for obesity. One hundred and sixty-six (81.8\%) respondents were familiar with indications for a bariatric oper- 
Table I. Physicians' voivodeship of residence

\begin{tabular}{|c|c|c|c|c|}
\hline Voivodeship & Physicians, $n$ (\%) & Male, $n(\%)$ & Female, $n(\%)$ & Age [years] \\
\hline Małopolskie & $44(21.36)$ & $15(7.28)$ & $29(14.08)$ & $41.95 \pm 10.96$ \\
\hline Zachodniopomorskie & $26(12.62)$ & $6(2.91)$ & $20(9.71)$ & $51.19 \pm 9.1$ \\
\hline Lubelskie & $23(11.17)$ & $6(2.91)$ & $17(8.25)$ & $48.48 \pm 9.66$ \\
\hline Dolnośląskie & $18(8.74)$ & $7(3.40)$ & $11(5.34)$ & $44.83 \pm 10.7$ \\
\hline Kujawsko-Pomorskie & $18(8.74)$ & $7(3.40)$ & $11(5.34)$ & $42.06 \pm 6.81$ \\
\hline Mazowieckie & $15(7.28)$ & $5(2.43)$ & $10(4.85)$ & $48.73 \pm 11.47$ \\
\hline Łódzkie & $14(6.80)$ & $9(4.37)$ & $5(2.43)$ & $42.21 \pm 10.84$ \\
\hline Podlaskie & $9(4.37)$ & $1(0.49)$ & $8(3.88)$ & $52.78 \pm 10.06$ \\
\hline Śląskie & $9(4.37)$ & 0 & $9(4.37)$ & $39.78 \pm 11.26$ \\
\hline Podkarpackie & $7(3.40)$ & $2(0.97)$ & $5(2.43)$ & $48.14 \pm 6.54$ \\
\hline Pomorskie & $5(2.43)$ & $3(1.46)$ & $2(0.97)$ & $57.2 \pm 9.81$ \\
\hline Lubuskie & $4(1.94)$ & $1(0.49)$ & $3(1.46)$ & $41.25 \pm 12.04$ \\
\hline Warmińsko-Mazurskie & $4(1.94)$ & $3(1.46)$ & $1(0.49)$ & $61 \pm 17.73$ \\
\hline Wielkopolskie & $3(1.46)$ & 0 & $3(1.46)$ & $55.67 \pm 4.04$ \\
\hline Świętokrzyskie & $1(0.49)$ & 0 & $1(0.49)$ & 42 \\
\hline Not declared & $6(2.91)$ & $2(0.97)$ & $4(1.94)$ & $54.67 \pm 8.31$ \\
\hline Total & $206(100)$ & $67(32.52)$ & $139(67.48)$ & $46.39 \pm 10.87$ \\
\hline
\end{tabular}

ation. One hundred and fifty-four (75.5\%) physicians declared that they are able to explain to their patients how bariatric operations are performed, but only 141 (69.8\%) respondents could name the most commonly performed bariatric procedures. The fact that bariatric surgery in Poland is financed by the National Health Fund was known to $168(82.4 \%)$ doctors answering the survey. The great majority of respondents, 198 (96.6\%), were aware that bariatric surgery is efficient in the treatment of the metabolic syndrome. Additionally, most of physicians $(189-93.6 \%)$ who took part in the survey knew that metabolic surgery can be helpful in reduction of treatment cost for metabolic syndrome. Fifty-seven (27.8\%) doctors admitted they had never referred a patient to a bariatric surgeon (Table II). Answers to questions 13 and 14 showed a disproportion between the number of patients who would be potential candidates for bariatric treatment, who are currently under the care of participating physicians, and the number of patients who are being referred to a bariatric surgeon (Figure 1). Fifty-nine (28.8\%) physicians did not refer any patients for surgical treatment of obesity, but only $4(2 \%)$ claimed that they did not have any patient who would meet the eligibility criteria for a bariatric operation. One hundred and twenty-five (63\%) physicians refer $1-4$ patients a year to a bariatric surgeon, but 56 (28\%) declared that they have 1-4 patients eligible for bariatric surgery. Thirteen (6.6\%) doctors claimed that they send 5-19 patients a year for a bariatric operation, but 102 (51\%) stated that they have 5-19 patients qualifying for the surgical treatment of obesity. Only 1 (0.5\%) doctor reported referring more than 20 patients a year for a bariatric operation, but 23 (11.5\%) physicians answered that they have 20-49 patients currently under their care. None of the participating physicians claimed to refer 50 or more patients a year for bariatric surgery. Twenty-three percent of physicians answered that they have 20-49 candidates for bariatric surgery in their practice and 15 (7.5\%) claimed to have more than 50 . One hundred and eighty-seven (90.8\%) respondents responded positively when asked if they would like to participate in training designed for general practitioners of which 
Table II. Results of the survey

\begin{tabular}{|lcc|}
\hline Question & Yes, $n$ (\%) & No, $n$ (\%) \\
\hline Have you heard about the possibility of surgical treatment of obesity? & $204(99.5)$ & $1(0.5)$ \\
\hline Do you know the indications for a bariatric operation? & $166(81.8)$ & $37(18.2)$ \\
\hline Could you name the most popular bariatric procedures? & $141(69.8)$ & $61(30.2)$ \\
\hline Could you explain to your patient how the most common bariatric procedures are conducted? & $154(75.5)$ & $50(24.5)$ \\
\hline Do you think that bariatric operations are reimbursed by the National Health Fund? & $168(82.4)$ & $36(17.6)$ \\
\hline Do you think that bariatric procedures may help in the treatment of metabolic syndrome? & $198(96.6)$ & $7(3.4)$ \\
\hline $\begin{array}{l}\text { Do you think that bariatric surgery may allow the costs of treating patients with metabolic } \\
\text { syndrome to be reduced? }\end{array}$ & $189(93.6)$ & $13(6.4)$ \\
\hline Have you ever referred your patient to a bariatric surgeon for surgical treatment of obesity? & $148(72.2)$ & $57(27.8)$ \\
\hline $\begin{array}{l}\text { Would you be willing to participate in training designed for general practitioners, whose topic } \\
\text { would be associated with the surgical treatment of obesity? }\end{array}$ & $187(90.8)$ & $19(9.2)$ \\
\hline
\end{tabular}

the main topic would be associated with the surgical treatment of obesity.

\section{Discussion}

Bariatric surgery is quickly gaining popularity in Poland, mainly due to increasing obesity prevalence. According to a study by Janik et al., from 2007 to 2014 , the total number of bariatric procedures per year increased from 176 in 2007 to 1499 in 2014 in surgical departments which answered their survey. Among them, $96.4 \%$ were performed laparoscopically. The most commonly reported procedures in 2014 were LSG (60.7\%) and LRYGB (19.2\%) [16]. Knowledge regarding bariatric surgery among Polish general practitioners seems to have been increasing since 2012, when Giaro et al. conducted a study in which only $8.1 \%$ of Polish GPs participating in the survey had theoretical, practical and 'epidemiological consciousness' concerning surgical treatment of obesity [20]. We expected an increase in knowledge about bariatrics between 2012 and 2016, because of the ongoing education of the physicians. This is a result of publications in popular medical journals, conferences concerning bariatric surgery in Poland and activity of the patients' support groups. However, the questionnaire given to surgeons in 2014 yielded disappointing results. Only $25 \%$ participating in the survey indicated theoretical, practical and epidemiological knowledge concerning surgical treatment of obesity [22]. A study from 2012-2013 evaluating knowledge of Polish students about the surgical treatment of obesity showed that it was low and the

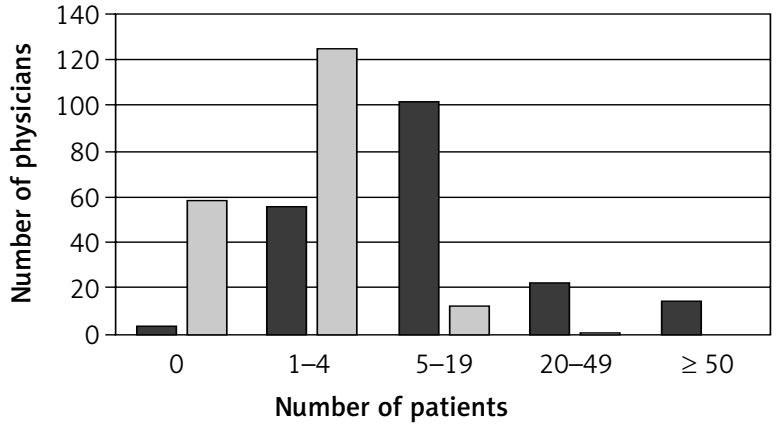

Question 13. How many patients currently do you have under your care, who would qualify for bariatric surgery?

$\square$ Question 14. How many patients do you refer to a bariatric surgeon for surgical treatment of obesity a year?

Figure 1. Results of the survey: answers to question number 13-14

curriculum at Polish medical schools does not pay sufficient attention to metabolic surgery [23]. Our current results show that physicians are almost always (99.5\%) familiar with the possibility of surgical treatment of obesity, but only 166 (81.8\%) respondents knew the indications for a bariatric operation. In the study published by Giaro et al. the only surgical technique of treatment for obesity known by $39 \%$ of participating GPs was the gastric balloon implantation [20], and in the study by Auspitz et al. only $23.8 \%$ of physicians felt comfortable explaining the procedure to the patient [24]. In our study $75.5 \%$ of physicians were able to explain to their patients how the bariatric operations are performed, and 69.8\% of respondents could name the most commonly performed bariatric procedures. Tork et al. published in 
2015 results of a survey presented to primary care physicians which indicated that all respondents were aware of the benefits of bariatric surgery, including diminishing diabetes, hypertension and hyperlipidemia [21]. A publication by Auspitz et al. showed that $48.1 \%$ of physicians responding to the survey support metabolic surgery for patients with diabetes [24]. Our results showed that the majority of respondents $(96.6 \%)$ were aware that bariatric surgery is useful in the treatment of metabolic syndrome. Also most of the physicians (93.6\%) agree that metabolic surgery can help to reduce treatment costs for syndrome X. The survey by Tork et al. showed that most respondents (59\%) reported that at least 1 in 4 of their patients had a BMI $\geq 35 \mathrm{~kg} / \mathrm{m}^{2}$, but only $9 \%$ indicated that they frequently or almost always refer their morbidly obese patients to bariatric surgeons [21]. In a study by Auspitz et al., $87 \%$ of physicians had referred patients for bariatric surgery, but most (70.2\%) had referred no more than $5 \%$ of their morbidly obese patients within the previous 12 months [24]. We found that 59 physicians participating in our study did not refer any patients to bariatric surgery but only 4 claimed that they did not have any candidates for a bariatric operation. Most of the participants $(63.1 \%)$ stated that they send $1-4$ patients a year to a bariatric surgeon, but also a large number of doctors (51\%) declared they had 5-19 candidates for the surgical treatment of obesity. Results obtained by Tork et al. showed that $63 \%$ of physicians who answered the survey believed that providing educational pamphlets and seminars to GPs on the topic of treatment and management of morbidly obese patients would increase referrals to bariatric surgery [21]. In the publication by Auspitz et al., $92.5 \%$ of respondents would like to receive more education about bariatric surgery [24]. A previous Polish survey determined that nearly $92 \%$ of respondents see the need for education, regarding the surgical treatment of obesity [20]. Also a great majority of physicians in our study $(90.8 \%)$ would like to learn more about surgical treatment of obesity. In conclusion, the knowledge concerning bariatric surgery among Polish GPs is improving, but it still needs to be expanded.

\section{Conclusions}

Our study demonstrates that nowadays bariatric surgery is a recognized method of treatment, but physicians remain reluctant to refer their patients to surgical treatment of obesity. It was found that there is a large disproportion between the number of patients being referred to a bariatric surgeon and the number of patients who require this treatment. It may be a result of lack of knowledge concerning indications for metabolic surgery, principles of management for patients before and after a bariatric procedure or unawareness that this treatment would be reimbursed by the National Health Fund. However, GPs declare willingness to learn more about this method, which is frequently missed out in the course of their medical education.

\section{Acknowledgments}

The publication of this article was supported by the Faculty of Medicine, Jagiellonian University Medical College, Leading National Research Centre (KNOW) 2012-2017.

\section{Conflict of interest}

The authors declare no conflict of interest.

\section{References}

1. WHO. WHO Obesity and overweight. World Health Organisation Media Centre Fact Sheet No. 311, 2012.

2. Mitchell J, Catenacci N, Wyatt V, Hill HR. Obesity: overview of an epidemic. Psychiatr Clin North Am 2011; 34: 717-32.

3. Rosiek A, Maciejewska NF, Leksowski K, et al. Effect of television on oesity and excess of weight and consequences of health. Int J Environ Res Public Health 2015; 12: 9408-26.

4. Pischon T, Boeing H, Hoffmann K. General and abdominal adiposity and risk of death in Europe. N Engl J Med 2008; 359: 2105-20.

5. Haslam DW, James WPT. Obesity. Lancet 2005; 366: 1197-209.

6. Li Z, Bowerman S, Heber D. Health ramifications of the obesity epidemic. Surg Clin North Am 2005; 85: 681-701.

7. Mitura K, Garnysz K. In search of the ideal patient for the intragastric balloon - short- and long-term results in 70 obese patients. Videosurgery Miniinv 2015; 10: 541-7.

8. Hoelscher DM, Kirk S, Ritchie L, Cunningham-Sabo L. Position of the Academy of Nutrition and Dietetics: interventions for the prevention and treatment of pediatric overweight and obesity. J Acad Nutr Diet 2013; 113: 1375-94.

9. Dumon K, Savulionyte G. Bariatric surgery produces greater weight loss and improvements in medical conditions than non-surgical treatment of obesity. Evid Based Med 2014; 19: 138.

10. Gloy VL, Briel M, Bhatt $D L$, et al. Bariatric surgery versus non-surgical treatment for obesity: a systematic review and meta-analysis. BMJ 2013; 5934: 1-16.

11. Morshed G, Fathy SM. Impact of post-laparoscopic sleeve gastrectomy weight loss on C-reactive protein, lipid profile and 
CA-125 in morbidly obese women. Videosurgery Miniinv 2015; 10: $521-6$.

12. Ersoz F, Duzkoylu Y, Deniz MM, Boz M. Laparoscopic Roux-en-Y gastric bypass with ileal transposition - an alternative surgical treatment for type 2 diabetes mellitus and gastroesophageal reflux. Videosurgery Miniinv 2015; 10: 481-5.

13. Major P, Matłok M, Pędziwiatr M, et al. Quality of life after bariatric surgery. Obes Surg 2015; 25: 1703-10.

14. McCarty TM. Can bariatric surgery be done as an outpatient procedure? Adv Surg 2006; 40: 99-106.

15. Clough A, Hamill D, Jackson S, et al. Outcome of three common bariatric procedures in the public sector. ANZ J Surg 2016; doi: 10.1111/ans.13585.

16. Janik MR, Stanowski E, Paśnik K. Present status of bariatric surgery in Poland. Videosurgery Miniinv 2016; 11: 22-5.

17. Brown WA, Burton PR, Shaw K, et al. A pre-hospital patient education program improves outcomes of bariatric surgery. Obes Surg 2016; 26: 2074-81.

18. Shannon C, Gervasoni A, Williams T. The bariatric surgery patient: nutrition considerations. Aust Fam Physician 2013; 42: 547-52.

19. Paolino L, Genser L, Fritsch S, et al. The web-surfing bariatic patient: the role of the internet in the decision-making process. Obes Surg 2015; 25: 738-43.

20. Giaro M, Wyleżoł M, Truszczyński O, Lewandowski T. Assess ment of the knowledge of GPS considering the surgical treatment of obesity. Pol Przegl Chir 2012; 84: 383-9.

21. Tork S, Meister KM, Uebele AL, et al. Factors influencing primary care physicians' referral for bariatric surgery. JSLS 2015; 19: pii: e2015.00046.

22. Giaro M, Wyleżoł MS, Lipski P, Truszczyński O. An evaluation of the knowledge of the surgical treatment of obesity among surgeons. Videosurgery Miniinv 2014; 9: 6-12.

23. Matłok M, Pędziwiatr M, Major P, et al. The knowledge of Polish medical students about surgical treatment of obesity. Eur Surg 2015; 47: 266-70.

24. Auspitz M, Cleghorn MC, Azin A, et al. Knowledge and perception of bariatric surgery among primary care physicians: a survey of family doctors in Ontario. Obes Surg 2016; 26: 2022-8.

Received: 6.07.2016, accepted: 24.08.2016. 
Appendix. Multiple-choice questionnaire

Survey:

1. Gender:

- Male

- Female

2. Age:

- [number]

3. Specialization:

- Family medicine

- Pediatrics

- Internal medicine

- Other

4. Voivodeship:

- Dolnośląskie

- Kujawsko-Pomorskie

- Lubelskie

- Lubuskie

- Łódzkie

- Małopolskie

- Mazowieckie

- Opolskie

- Podkarpackie

- Podlaskie

- Pomorskie

- Śląskie

- Świętokrzyskie

- Warmińsko-Mazurskie

- Wielkopolskie

- Zachodniopomorskie

5. Have you heard about the possibility of surgical treatment of obesity?

- Yes

- No

6. Do you know the indications for a bariatric operation?

- Yes

- No
7. Could you name the most popular bariatric procedures?

- Yes

- No

8. Could you explain to your patient how the most common bariatric procedures are conducted?

- Yes

- No

9. Do you think that bariatric operations are reimbursed by the National Health Fund?

- Yes

- No

10. Do you think that bariatric procedures may help in the treatment of metabolic syndrome?

- Yes

- No

11. Do you think that bariatric surgery may allow the costs of treating patients suffering from metabolic syndrome to be reduced?

- Yes

- No

12. Have you ever referred your patient to a bariatric surgeon for surgical treatment of obesity?

- Yes

- No

13. Could you estimate how many patients you refer to a bariatric surgeon for surgical treatment of obesity per year?

- [number]

14. Could you estimate how many patients you currently have under your care, who would qualify for bariatric surgery?

- [number]

15. Would you be willing to participate in training designed for general practitioners, whose theme would be associated with the surgical treatment of obesity?

- Yes

- No 